\title{
Os Grandes Registros Brasileiros de Intervenção Coronária Percutânea
}

\author{
Áurea J. Chaves
}

$\mathrm{H}$ menos de dois anos, a Revista Brasileira de Cardiologia Invasiva (RBCI) vem passando por uma fase de inovação e expansão, que redundou em reconhecimento e aceitação crescentes entre os leitores, refletidos no aumento de mais de $3.000 \%$ de acessos a seu portal eletrônico verificado nesse período.

Esse reconhecimento tem sido um dos fatores responsáveis pela captação de artigos de grande interesse científico, antes destinados a outros periódicos da especialidade, nacionais e internacionais. Nesta edição fomos brindados com fato inédito: a submissão de registros de cinco dos maiores centros brasileiros de intervenção coronária percutânea.

O Registro DESIRE, do Hospital do Coração/Associação do Sanatório Sírio de São Paulo, SP, pioneiro, que reúne dados do maior número de pacientes tratados com stents farmacológicos e que vem contribuindo sistematicamente com a divulgação de seus resultados (follow-up de cinco anos, incidência e preditores de trombose dos stents, resultados em diabéticos), traz agora análise do manuseio de situação desafiadora, a reestenose dos stents farmacológicos. O Registro EINSTEIN, do Hospital Israelita Albert Einstein, e o Registro INCOR, do Instituto do Coração do Hospital das Clínicas da Universidade de São Paulo, ambos de São Paulo, SP, avaliam a utilização dos stents farmacológicos em pacientes com síndromes agudas, com e sem supradesnivelamento do segmento ST, respectivamente. Junta-se a eles o Registro da Clínica São Vicente, do Rio de Janeiro, RJ, que analisa o desempenho dos stents farmacológicos nas indicações de tratamento off-label. Complementa a lista desses grandes registros o Registro IC-FUC, do Instituto de Cardiologia-Fundação Universitária de Cardiologia, de Porto Alegre, RS, que examina a incidência e os preditores de complicações vasculares de via de acesso, freqüentemente negligenciados como causa de morbidade e mortalidade, em pacientes submetidos a intervenção coronária percutânea.

Temos também, nesta edição, outro registro, multicêntrico, que mostra uma prevalência não desprezível de estenoses da artéria subclávia esquerda, proximal à artéria torácica interna esquerda, que pode comprome- ter o desempenho desse enxerto, utilizado em mais de 90\% das cirurgias de revascularização miocárdica.

Serviços de Cardiologia Intervencionista mais novos, e com casuísticas menores, têm organizado seus bancos de dados e se destacado por apresentar estudos que analisam temas contemporâneos. A Santa Casa de Marília, SP, e o Hospital do Coração de Londrina, PR, trazem nesta edição dois artigos muito interessantes, que avaliam a técnica ulnar para procedimentos coronários e a cateterização direita e esquerda por via radial. É exemplo que esperamos seja seguido por outras instituições de características semelhantes e que desejem se destacar, não só do ponto de vista assistencial como também científico.

O Instituto Dante Pazzanese de Cardiologia, em São Paulo, SP, que sempre se sobressai por trazer artigos com resultados de estudos first-in-man em intervenções percutâneas, traz artigos que analisam o desempenho dos stents dedicados ao tratamento de lesões em vasos de fino calibre e pontes de safena. Um desses estudos, a avaliação dos resultados de um stent auto-expansível de baixíssimo perfil, com maior potencial de navegabilidade que os stents usuais e que permite acesso aos vasos de mais fino calibre, é tema de editorial dos drs. Hiram Bezerra e Marco Costa, da Case Western Reserve University, de Cleveland, Estados Unidos. Já os resultados de outro estudo que compara dois stents diferentes, recobertos com redes protetoras para evitar embolização distal, no tratamento de lesões em pontes de safena são analisados com bastante equilíbrio e sensatez pelo dr. Hugo Londero, do Sanatório Allende, em Córdoba, Argentina.

Da América Latina recebemos nova contribuição, o estudo REMCLI-ATC, realizado no Hospital de Clínicas de Montevidéu, Uruguai, que avalia de maneira randomizada a realização de angioplastias coronárias com materiais novos ou reprocessados.

Trouxemos ainda artigo de revisão que aborda o tema "Dissecção da Artéria Carótida", que despertou muito interesse recentemente, explorando em profundidade as mais modernas técnicas diagnósticas da ressonância magnética e da tomografia computadorizada, essenciais no diagnóstico e manuseio dessa condição. 
Para finalizar, gostaríamos de fazer agradecimento especial aos drs. Fabio B. Jatene, do Instituto do Coração, e Luiz Alberto Mattos, do Instituto Dante Pazzanese de Cardiologia, de São Paulo, SP, que, recém-chegados de congressos europeus de suas especialidades e inspirados por notáveis cavaleiros do Im- pério Britânico, trouxeram, respectivamente, as visões do cirurgião e do intervencionista a respeito do estudo SYNTAX, que avalia os resultados das duas modalidades de tratamento na doença coronária complexa.

Áurea J. Chaves Editora 